\title{
VARIABILITY, TRAITS ASSOCIATION AND PATH COEFFICIENT OF YIELD AND YIELD CONTRIBUTING TRAITS OF SELECTED BORO RICE (ORYZA SATIVA L.) GERMPLASM
}

\author{
T. Chakrabarty ", M.Z. Islam, N. Akter and M. Khalequzzaman \\ Genetic Resources and Seed Division, Bangladesh Rice Research Institute \\ Gazipur, Bangladesh
}

\begin{abstract}
Boro season is the most productive among Aus, Aman and Boro seasons. For confirming food security, we need to emphasize more on Boro season to feed more people. That's why forty entries of Boro rice germplasm were allowed to evaluate for their genetic variations in yield and yield contributing traits, their associations and also direct and indirect effects on yield. Observing means, range and all genetic parameters, selection could be done on the basis of number of effective tillers, harvest index, filled grains per panicle, unfilled grains per panicle, days to maturity and grain yield. Traits association analysis revealed that days to flowering, days to maturity and filled grain per panicle could significantly improve rice germplasm. Path analysis estimated that direct selection based on days to flowering, days to maturity and filled grain per panicle would be efficient for improvement of rice germplasm. G5 had the highest yield along with filled grain per plant, harvest index and 1000 grain weight and it could be used as potential variety for improving yield. The germplasm G7, G19, G9 and G33 could be used in further breeding program for the improvement of rice germplasm.
\end{abstract}

Keywords: Germplasm, Variability, Trait association, Path coefficient analysis

\section{INTRODUCTION}

Rice (Oryza sativa L.) is one of the most important and top prioritized crop for many nations in the world, which is under the family Poaceae. Bangladesh is one of the highest rice growing country in the world as well as the staple food of this country, this state needs profuse advancement in quality breeding lines to meet up the upcoming population hunger. The cultivable lands are decreasing day by day due to industrialization (Mahmud, 2003). Bangladeshi rice cultivation heavily depends on

\footnotetext{
*Corresponding author: tonmoyarani@gmail.com
} 
Aman season due to rainfed condition but the yield performance is significantly lower than the Boro season (Williams et al., 2006). It has been estimated that the world will have to produce $60 \%$ more rice by 2030 than what it produced in 1995 (Babu et al., 2012). To meet up the hunger we need to emphasize on Boro rice production so that the sustainable food security will be achieved by Bangladesh. Though Boro rice is long duration but it is balanced by the yield. But it is crucial to find out the marvelous breeding lines having short duration without compromising with yield for Boro season. Though yield is a polygenic trait so it is governed by the many genes and having a complex association with many yield contributing characters. Those characters are profusely influenced by the environmental factors (Doehlert et al., 2001).

A complete, efficient and meaningful breeding program will led to find out more genetic variation, selection of genotypes and advancement of selected genotypes for the varietal development (Ye et al., 2013, Peyman; 2012 and Chakravorty et al., 2013). Heritability estimation helps to find out the flow of traits from parents to offspring. Heritability alone can't be used in selection criteria also requires genetic advance for efficacy.

This study was undertaken to find out the variability among the traits of selected Boro germplasm, association between the traits and yield to find out the selection guidelines, path coefficient among the traits with yield for the effective selection and to find out the promising genotypes for the further advancement of the rice breeding.

\section{MATERIALS AND METHODS}

\section{Materials}

All forty entries of Boro rice germplasm (Table 1) were collected from the genebank of Bangladesh Rice Research Institute (BRRI). The germplasm were sown in the seedbed in 30 November 2016 and raised 40 days seedling in the Genetic Resources and Seed Division farm of BRRI. The soil was slightly acidic ( $\mathrm{pH} 6.5)$, silt clay and low in organic matter $(0.86 \%)$, having sharp differences in summer and winter temperatures. Hence the seedlings were transplanted in the main experimental field with one seedling per hill in 9 January 2017. The row to row and plant to plant distance was maintained $20 \mathrm{~cm} \times 20 \mathrm{~cm}$. There were 30 hills for each germplasm in each replication. Entries were repeated for three times. The experiment was laid out in a Randomized Complete Block Design (RCBD). The recommended agronomic practices were maintained for the proper crop growth. 
Table 1. Selected Boro germplasm from BRRI genebank

\begin{tabular}{lllllllllll}
\hline S1. N & $\begin{array}{c}\text { Germplasm } \\
\text { Names }\end{array}$ & S1. N & $\begin{array}{c}\text { Germplasm } \\
\text { Names }\end{array}$ & S1. N & $\begin{array}{c}\text { Germplasm } \\
\text { Names }\end{array}$ & S1. N & $\begin{array}{c}\text { Germplasm } \\
\text { Names }\end{array}$ \\
\hline G1. & Banajira & G11. & Jagli & G21. & Boro 398 & G31. & Chinese var\#1 \\
G2. & Ghuniboro & G12. & Boro & G22. & Boro 465 & G32. & Firooz \\
G3. & Aushaboro & G13. & Tupa & G23. & Boro 522 & G33. & Moghalsail \\
G4. & Kaikka boro & G14. & Sadaboro & G24. & Boro259 & G34. & Fenaful \\
G5. & Khamarang & G15. & Sailboro & G25. & Dhaliboro7/2 & G35. & Bichi barui \\
G6. & Deshi boro & G16. & Birion & G26. & Dhaliboro87/1 & G36. & Toifaboro \\
G7. & Jamir & G17. & Boro 66/1 & G27. & Dhali boro94 & G37. & Biroin \\
G8. & Isamoti & G18. & Boro69/2 & G28. & Dhaliboro104/1 & G38. & Chengri \\
G9. & Sadaboro & G19. & Boro135/1 & G29. & Dhaliboro & G39. & Unknown \\
G10. & Bogra & G20. & Boro 391 & G30. & Omniboro & G40. & Sonaliboro2 \\
\hline
\end{tabular}

\section{Data collection}

Data were collected from the randomly selected ten plants in each replication for plant height $(\mathrm{cm})$, flag leaf length $(\mathrm{cm})$, flag leaf width $(\mathrm{cm})$, days to flowering, days to maturity, panicle length $(\mathrm{cm})$, effective tiller number, harvest index, filled grains per panicle, unfilled grain per panicle, grain length $(\mathrm{mm})$, grain breadth/width $(\mathrm{mm})$, 1000 grain weight $(\mathrm{g})$ and yield $\left(\mathrm{kg} \mathrm{ha}^{-1}\right)$.

\section{Statistical analysis}

Mean, range and standard deviation (SD) for each character were estimated. The mean sum of square was estimated for the calculation of genotypic and phenotypic variances (Johnson et al., 1955). Genotypic and phenotypic coefficients of variation were estimated by the formula suggested by Burton (1952). Heritability in broad sense was estimated which was further defined by Lush (1949) by the suggested formula (Johnson et al., 1955). The expected genetic advance for different characters was calculated using the suggested formula (Johnson et al., 1955 and Lush, 1949). Genetic advance in percentage of mean was calculated by the given formula (Comstock et al., 1952). Phenotypic and genotypic correlations were worked out by using the suggested formulae (Falconer, 1964). Analysis of path coefficient suggested (Wright, 1921) and modified by (Dewey and Lu, 1959) was used to calculate the direct and indirect contribution of various traits to yield.

\section{RESULTS AND DISCUSSION}

The mean analysis given in table 2 shows that twenty seven (27) genotypes for plant height, twenty four (24) genotypes for flag leaf length, twenty five (25) genotypes for 
flag leaf width, sixteen (16) genotypes for days to flowering, nineteen (19) genotypes for days to maturity, nineteen (19) genotypes for panicle length, thirteen (13) genotypes for effective tiller number, twenty two (22) genotypes for harvest index, seventeen (17) genotypes for filled grain per panicle, ten (10) genotypes for unfilled grain per panicle, nineteen (19) genotypes for grain length, twenty one (21) genotypes for grain breadth, twenty two (22) genotypes for 1000 grain weight and twenty (20) genotypes for yield/hill performed over their grand mean. The highest plant height was found in G13 followed by G24, G34 and G35. The highest flag leaf length was found in G21 followed by G11, G20 and G30. The highest flag leaf width was found in G11 followed by G12, G13 and G29. The highest days to flowering was found in G29 followed by G5, G30 and G7. The highest days to maturity was found in G5 followed by G24, G25 and G34. The highest panicle length was found in G34 followed by G24, G32 and G33. The highest effective tiller number was found in G6 followed by G9, G1 and G33. The highest harvest index was found in G3 followed by G35, G19 and G10. The highest filled grain per panicle was found in G34 followed by G5, G24, G33 and G11. The highest unfilled grain per panicle was found in G26 followed by G32 and G34. The highest grain length was found in G32 followed by G39, G9 and G31. The highest grain breadth was found in G8 followed by G22, G7, G20 and G23. The highest 1000 grain weight was found in G32 followed by G8, G31 and G5. The highest yield was found in G5 followed by G7, G19, G9 and G33.

Table 2. Mean performance of selected 40 Boro rice germplasm

\begin{tabular}{l|l|l|l|l|l|l|l}
\hline Variety & $\begin{array}{c}\text { Plant } \\
\text { Height }(\mathrm{cm})\end{array}$ & $\begin{array}{c}\text { Flag Leaf } \\
\text { Length }(\mathrm{cm})\end{array}$ & $\begin{array}{c}\text { Flag Leaf } \\
\text { Width }(\mathrm{cm})\end{array}$ & $\begin{array}{c}\text { Days to } \\
\text { Flowering }\end{array}$ & $\begin{array}{c}\text { Days to } \\
\text { Maturity }\end{array}$ & $\begin{array}{c}\text { Panicle } \\
\text { Length(cm) }\end{array}$ & $\begin{array}{c}\text { Effective } \\
\text { Tillers }\end{array}$ \\
\hline G1 & 102.07 & 26.77 & 0.90 & 119.00 & 147.33 & 19.37 & 14.00 \\
G2 & 92.53 & 28.77 & 1.06 & 112.00 & 141.33 & 18.27 & 9.33 \\
G3 & 89.77 & 28.50 & 0.96 & 111.00 & 141.67 & 17.70 & 11.00 \\
G4 & 85.60 & 29.60 & 1.01 & 112.00 & 142.33 & 17.17 & 11.00 \\
G5 & 54.40 & 32.97 & 1.03 & 130.00 & 163.00 & 14.70 & 10.33 \\
G6 & 104.03 & 22.60 & 0.81 & 121.00 & 148.67 & 17.67 & 26.00 \\
G7 & 106.90 & 32.10 & 1.03 & 124.33 & 143.33 & 21.23 & 9.67 \\
G8 & 107.77 & 30.37 & 1.04 & 118.33 & 147.00 & 20.53 & 12.00 \\
G9 & 89.00 & 26.63 & 0.88 & 117.33 & 145.67 & 19.17 & 19.00 \\
G10 & 99.40 & 32.97 & 1.05 & 116.67 & 145.67 & 18.87 & 12.33 \\
G11 & 113.47 & 38.43 & 1.22 & 115.00 & 144.33 & 20.63 & 9.33 \\
G12 & 105.23 & 37.60 & 1.14 & 115.33 & 147.33 & 21.43 & 8.00 \\
G13 & 124.27 & 34.27 & 1.14 & 120.67 & 149.33 & 23.43 & 12.33 \\
G14 & 106.20 & 35.73 & 1.12 & 114.33 & 144.00 & 21.80 & 9.00 \\
G15 & 115.20 & 36.00 & 1.12 & 116.00 & 145.00 & 20.93 & 11.33
\end{tabular}




\begin{tabular}{|c|c|c|c|c|c|c|c|}
\hline Variety & \begin{tabular}{c|} 
Plant \\
Height $(\mathrm{cm})$
\end{tabular} & $\begin{array}{c}\text { Flag Leaf } \\
\text { Length }(\mathrm{cm})\end{array}$ & $\begin{array}{c}\text { Flag Leaf } \\
\text { Width }(\mathrm{cm})\end{array}$ & \begin{tabular}{|c|} 
Days to \\
Flowering
\end{tabular} & $\begin{array}{l}\text { Days to } \\
\text { Maturity }\end{array}$ & $\begin{array}{c}\text { Panicle } \\
\text { Length }(\mathrm{cm})\end{array}$ & \begin{tabular}{|c|} 
Effective \\
Tillers
\end{tabular} \\
\hline$\overline{\text { G16 }}$ & 105.87 & 35.90 & 1.06 & 114.67 & 144.00 & 20.87 & 11.33 \\
\hline G17 & 114.50 & 36.63 & 1.05 & 115.67 & 144.00 & 20.50 & 10.33 \\
\hline G18 & 111.17 & 36.73 & 1.07 & 116.00 & 145.33 & 20.17 & 10.67 \\
\hline G19 & 105.30 & 34.97 & 1.10 & 117.67 & 146.00 & 19.50 & 10.67 \\
\hline G20 & 109.17 & 38.30 & 1.13 & 113.33 & 142.00 & 20.47 & 11.33 \\
\hline G21 & 117.73 & 38.60 & 1.12 & 117.33 & 146.00 & 21.87 & 10.00 \\
\hline G22 & 105.50 & 35.07 & 1.12 & 113.67 & 143.00 & 20.90 & 10.00 \\
\hline G23 & 115.13 & 36.13 & 1.13 & 118.00 & 147.00 & 22.27 & 8.33 \\
\hline G24 & 122.13 & 32.67 & 1.13 & 123.00 & 153.33 & 24.63 & 11.00 \\
\hline G25 & 118.47 & 28.43 & 1.07 & 121.33 & 151.67 & 23.17 & 13.00 \\
\hline G26 & 112.67 & 37.77 & 1.09 & 116.67 & 148.33 & 20.10 & 12.67 \\
\hline G27 & 108.90 & 33.80 & 1.03 & 119.33 & 148.33 & 20.10 & 11.00 \\
\hline G28 & 111.53 & 34.00 & 1.10 & 117.33 & 146.00 & 21.00 & 11.00 \\
\hline G29 & 115.70 & 37.50 & 1.14 & 131.33 & 148.67 & 22.03 & 10.67 \\
\hline G30 & 104.93 & 38.13 & 1.12 & 128.00 & 146.00 & 19.50 & 10.00 \\
\hline G31 & 45.47 & 18.67 & 1.08 & 110.67 & 139.33 & 16.40 & 8.00 \\
\hline G32 & 100.83 & 28.73 & 1.08 & 121.00 & 149.33 & 24.27 & 8.33 \\
\hline G33 & 105.10 & 29.93 & 1.02 & 119.67 & 147.00 & 23.40 & 14.00 \\
\hline G34 & 123.33 & 34.37 & 1.07 & 122.67 & 150.33 & 27.40 & 9.00 \\
\hline G35 & 120.30 & 29.70 & 1.06 & 118.33 & 146.33 & 23.00 & 7.67 \\
\hline G36 & 112.37 & 34.30 & 1.16 & 121.00 & 148.67 & 22.73 & 10.33 \\
\hline G37 & 107.87 & 34.07 & 1.11 & 114.33 & 142.33 & 20.10 & 9.33 \\
\hline G38 & 76.82 & 34.30 & 1.04 & 115.67 & 144.00 & 20.57 & 9.67 \\
\hline G39 & 107.20 & 32.20 & 0.99 & 113.33 & 142.00 & 20.60 & 11.67 \\
\hline G40 & 98.53 & 27.43 & 0.93 & 119.00 & 147.67 & 19.80 & 11.00 \\
\hline Grand Mean 1 & 104.31 & 32.79 & 1.06 & 118.05 & 146.32 & 20.71 & 11.14 \\
\hline $\begin{array}{l}\text { Coefficient } \\
\text { of variation }\end{array}$ & 13.01 & 8.46 & 4.62 & 4.63 & 1.55 & 7.99 & 6.23 \\
\hline $\begin{array}{l}\text { Standard } \\
\text { deviation }\end{array}$ & 16.26 & 4.52 & 0.08 & 4.73 & 4.03 & 2.34 & 3.15 \\
\hline $\begin{array}{l}\text { Standard } \\
\text { error }\end{array}$ & 7.84 & 1.60 & 0.03 & 3.15 & 1.31 & 0.95 & 0.40 \\
\hline $\begin{array}{l}\text { Critical } \\
\text { difference } \\
(0.05)\end{array}$ & 31.94 & 6.53 & 0.11 & 12.86 & 5.33 & 3.89 & 1.63 \\
\hline
\end{tabular}


Table 2. Mean performance of selected 40 Boro rice germplasm (Continue)

\begin{tabular}{|c|c|c|c|c|c|c|c|}
\hline Variety & $\begin{array}{c}\text { Harvest } \\
\text { Index }(\%)\end{array}$ & $\begin{array}{c}\text { Filled } \\
\text { Grain/Panicle }\end{array}$ & $\begin{array}{c}\text { Unfilled } \\
\text { Grain/Panicle }\end{array}$ & $\begin{array}{c}\text { Grain } \\
\text { Length }(\mathrm{mm})\end{array}$ & $\begin{array}{c}\text { Grain } \\
\text { Bredth }(\mathrm{mm})\end{array}$ & $\begin{array}{c}1000 \text { grain } \\
\text { weight }(\mathrm{g})\end{array}$ & $\begin{array}{c}\text { Yield } \\
\left(\begin{array}{c}\mathrm{kg} \mathrm{ha}^{-} \\
{ }^{-}\end{array}\right.\end{array}$ \\
\hline G1 & 26.26 & 56.33 & 1.00 & 7.86 & 3.10 & 20.59 & 2770.00 \\
\hline G2 & 36.27 & 53.33 & 2.67 & 7.76 & 3.04 & 18.75 & 1315.00 \\
\hline G3 & 49.93 & 46.67 & 2.33 & 7.77 & 2.81 & 18.38 & 1917.50 \\
\hline G4 & 29.87 & 59.00 & 2.33 & 7.76 & 3.19 & 19.56 & 2617.50 \\
\hline G5 & 37.61 & 107.33 & 9.00 & 8.14 & 2.95 & 20.85 & 3595.00 \\
\hline G6 & 28.16 & 43.67 & 2.33 & 7.40 & 3.03 & 16.81 & 2882.50 \\
\hline G7 & 33.69 & 56.33 & 6.33 & 7.92 & 3.24 & 19.99 & 3140.00 \\
\hline G8 & 28.37 & 58.33 & 3.33 & 8.06 & 3.27 & 21.30 & 1910.00 \\
\hline G9 & 36.16 & 65.67 & 2.33 & 8.25 & 3.13 & 20.83 & 2945.00 \\
\hline G10 & 43.68 & 49.00 & 3.33 & 7.55 & 3.06 & 18.90 & 2770.00 \\
\hline G11 & 35.86 & 81.00 & 4.00 & 7.75 & 3.17 & 20.41 & 2915.00 \\
\hline G12 & 36.12 & 64.00 & 3.33 & 7.76 & 2.99 & 19.10 & 2110.00 \\
\hline G13 & 27.37 & 75.33 & 3.00 & 7.82 & 2.83 & 16.36 & 2622.50 \\
\hline G14 & 27.97 & 80.33 & 5.67 & 7.83 & 3.03 & 19.39 & 2127.50 \\
\hline G15 & 43.21 & 70.67 & 1.33 & 7.66 & 3.03 & 19.87 & 2082.50 \\
\hline G16 & 32.48 & 55.67 & 2.67 & 7.69 & 3.02 & 19.02 & 2607.50 \\
\hline G17 & 35.47 & 64.00 & 4.33 & 7.96 & 3.22 & 19.85 & 2260.00 \\
\hline G18 & 40.52 & 76.33 & 3.33 & 7.90 & 3.23 & 18.56 & 2165.00 \\
\hline G19 & 46.19 & 61.00 & 4.33 & 7.61 & 3.21 & 21.34 & 3087.50 \\
\hline G20 & 37.05 & 69.00 & 3.33 & 7.93 & 3.23 & 19.05 & 1892.50 \\
\hline G21 & 38.32 & 70.33 & 4.33 & 7.68 & 3.09 & 16.87 & 2492.50 \\
\hline G22 & 40.45 & 61.33 & 3.67 & 7.71 & 3.26 & 20.04 & 2102.50 \\
\hline G23 & 33.47 & 47.67 & 2.67 & 7.94 & 3.23 & 20.42 & 2117.50 \\
\hline $\mathrm{G} 24$ & 25.44 & 82.67 & 4.67 & 7.94 & 3.11 & 17.43 & 2282.50 \\
\hline G25 & 28.76 & 67.00 & 3.00 & 7.70 & 2.90 & 17.73 & 2715.00 \\
\hline G26 & 26.85 & 36.67 & 30.67 & 7.73 & 3.22 & 19.90 & 1470.00 \\
\hline $\mathrm{G} 27$ & 32.81 & 66.00 & 5.67 & 8.18 & 2.99 & 20.36 & 1612.50 \\
\hline G28 & 36.43 & 60.33 & 9.00 & 7.84 & 3.13 & 20.33 & 1775.00 \\
\hline G29 & 34.53 & 75.00 & 4.33 & 7.75 & 3.04 & 18.40 & 2857.50 \\
\hline G30 & 40.88 & 69.00 & 4.00 & 7.56 & 3.13 & 18.40 & 1850.00 \\
\hline G31 & 36.97 & 51.33 & 4.67 & 8.25 & 2.91 & 20.86 & 1702.50 \\
\hline G32 & 32.34 & 59.67 & 15.67 & 9.74 & 2.76 & 21.40 & 1932.50 \\
\hline G33 & 39.28 & 81.00 & 4.33 & 7.24 & 2.77 & 16.67 & 2917.50 \\
\hline
\end{tabular}




\begin{tabular}{|c|c|c|c|c|c|c|c|}
\hline Variety & $\begin{array}{c}\text { Harvest } \\
\text { Index }(\%)\end{array}$ & $\begin{array}{c}\text { Filled } \\
\text { Grain/Panicle }\end{array}$ & $\begin{array}{c}\text { Unfilled } \\
\text { Grain/Panicle }\end{array}$ & $\begin{array}{c}\text { Grain } \\
\text { Length }(\mathrm{mm})\end{array}$ & $\begin{array}{c}\text { Grain } \\
\text { Bredth }(\mathrm{mm})\end{array}$ & $\begin{array}{l}1000 \text { grain } \\
\text { weight }(\mathrm{g})\end{array}$ & $\begin{array}{l}\text { Yield } \\
\left(\begin{array}{c}\mathrm{kg} \mathrm{ha}_{1} \\
\mathrm{~h}^{-}\end{array}\right.\end{array}$ \\
\hline G34 & 33.70 & 123.67 & 13.67 & 5.75 & 2.99 & 11.54 & 2342.50 \\
\hline G35 & 48.07 & 74.33 & 8.33 & 7.54 & 3.14 & 19.98 & 2687.50 \\
\hline G36 & 29.99 & 67.33 & 4.33 & 7.89 & 3.24 & 19.06 & 1947.50 \\
\hline G37 & 42.92 & 64.00 & 4.33 & 8.10 & 3.04 & 19.29 & 2877.50 \\
\hline G38 & 40.83 & 61.67 & 3.33 & 6.76 & 3.04 & 16.46 & 2725.00 \\
\hline G39 & 41.44 & 57.33 & 5.33 & 8.30 & 3.11 & 20.32 & 2755.00 \\
\hline G40 & 37.39 & 61.33 & 2.33 & 7.69 & 3.13 & 20.09 & 2732.50 \\
\hline $\begin{array}{l}\text { Grand } \\
\text { Mean }\end{array}$ & 35.83 & 65.77 & 5.22 & 7.79 & 3.07 & 19.11 & 2390.00 \\
\hline $\begin{array}{l}\text { Coefficient } \\
\text { of } \\
\text { variation }\end{array}$ & 7.89 & 2.97 & 13.00 & 4.67 & 5.40 & 6.46 & 7.95 \\
\hline $\begin{array}{l}\text { Standard } \\
\text { deviation }\end{array}$ & 6.14 & 15.81 & 5.07 & 0.53 & 0.14 & 1.85 & 516.56 \\
\hline $\begin{array}{l}\text { Standard } \\
\text { error }\end{array}$ & 1.63 & 1.23 & 0.39 & 0.21 & 0.09 & 0.71 & 109.71 \\
\hline $\begin{array}{l}\text { Critical } \\
\text { difference } \\
(0.05)\end{array}$ & 6.66 & 4.59 & 1.59 & 0.85 & 0.39 & 2.91 & 447.27 \\
\hline
\end{tabular}

In the table of variance and heritability (Table 3), high differences in genotypic and phenotypic variances were present for all the studied traits except for flag leaf width, effective tiller number, filled grain per panicle, yield and unfilled grain per panicle. It depicts that these traits are less influenced by environment. The highest genotypic and phenotypic coefficient of variation was found for unfilled grains per panicle (Islam et al., 2016). High heritability was observed for all traits except for plant height, flag leaf length, flag leaf width, days to flowering, days to maturity, panicle length, grain length, grain breadth and 1000 grain weight $(\mathrm{g})$. High heritability along with genetic advance (GA) and genetic advance in percent of mean (GAMP) was observed for effective tiller number, harvest index, filled grains per panicle, unfilled grains per panicle and grain yield. It means that these traits are controlled by multiple genes with high additive gene action and this result agreed with (Chuchert et al., 2016). 
Table 3. Variability analysis of selected 40 Boro rice germplasm

\begin{tabular}{l|l|l|l|l|l|l|l|l}
\hline & \multicolumn{1}{c}{ Range } & \multicolumn{1}{c}{$\mathrm{Vg}$} & $\mathrm{Vp}$ & $\mathrm{GCV}$ & $\mathrm{PCV}$ & $\mathrm{h}_{\mathrm{b}}{ }^{2}$ & $\mathrm{GA}$ & GAM $(\%)$ \\
\hline PH (cm) & $45.47-124.27$ & 203.07 & 387.27 & 13.66 & 18.87 & 52.44 & 16.30 & 15.63 \\
FLL(cm) & $18.67-38.6$ & 17.92 & 25.61 & 12.91 & 15.43 & 69.96 & 5.59 & 17.06 \\
FLW(cm) & $0.81-1.22$ & 0.01 & 0.01 & 7.21 & 8.57 & 70.79 & 0.10 & 9.58 \\
DF & $110.67-131.33$ & 12.50 & 42.36 & 2.99 & 5.51 & 29.51 & 3.03 & 2.57 \\
DM & $139.33-163$ & 14.54 & 19.68 & 2.61 & 3.03 & 73.89 & 5.18 & 3.54 \\
PL (cm) & $16.40-27.40$ & 4.60 & 7.34 & 10.36 & 13.08 & 62.72 & 2.68 & 12.96 \\
ET & $8.00-26.00$ & 9.74 & 10.22 & 28.02 & 28.70 & 95.29 & 4.81 & 43.21 \\
HI (\%) & $25.44-49.93$ & 35.06 & 43.06 & 16.53 & 18.31 & 81.42 & 8.44 & 23.56 \\
FG/P & $43.67-123.67$ & 248.79 & 252.60 & 23.98 & 24.17 & 98.49 & 24.73 & 37.61 \\
UFG/P & $1.00-30.67$ & 25.61 & 26.07 & 96.95 & 97.81 & 98.24 & 7.93 & 151.82 \\
GL (mm) & $5.75-9.74$ & 0.24 & 0.37 & 6.28 & 7.83 & 64.35 & 0.62 & 7.96 \\
GB (mm) & $2.76-3.27$ & 0.01 & 0.04 & 3.21 & 6.29 & 26.11 & 0.08 & 2.60 \\
1000 gwt. (g) & $11.54-21.40$ & 2.94 & 4.46 & 8.97 & 11.06 & 65.87 & 2.20 & 11.51 \\
Yield (kg/ha) & $1315-3595$ & 255008.67 & 291119.67 & 21.12 & 22.57 & 87.60 & 746.75 & 31.23 \\
\hline
\end{tabular}

$\mathrm{PH}=$ Plant height $(\mathrm{cm}), \mathrm{FLL}=$ Flag leaf length $(\mathrm{cm}), \mathrm{FLW}=$ Flag leaf width $(\mathrm{cm}), \mathrm{DF}=$ Days to flowering, $\mathrm{DM}=$ Days to maturity, $\mathrm{PL}=$ Panicle length $(\mathrm{cm}), \mathrm{ET}=$ Effective tiller, $\mathrm{HI}=$ Harvest index, $\mathrm{FG} / \mathrm{P}=\mathrm{No}$. of filled grain per plant, $\mathrm{UFG} / \mathrm{P}=$ No. of unfilled grain per plant, $\mathrm{GL}=$ Grain length $(\mathrm{mm}), \mathrm{GB}=\mathrm{Grain}$ breadth $(\mathrm{mm}), 1000$ gwt. $=1000$ grain weight $(\mathrm{g}), \mathrm{Vg}=$ Genotypic variance, $\mathrm{Vp}=$ Phenotypic variance, $\mathrm{GCV}=$ Genotypic coefficient of variation, $\mathrm{PCV}=$ Phenotypic coefficient of variation, $\mathrm{h}_{\mathrm{b}}{ }_{\mathrm{b}}=$ heritability, $\mathrm{GA}=$ Genetic advance, GAMP $=$ Genetic advance in percent of mean

The genotypic and phenotypic associations among selected traits are presented in Table 4. Genetic association value was always higher for all traits than their phenotypic ones. The positive and significant association was found for days to flowering, days to maturity and filled grain per panicle with yield/hill only in genotypic level and the result was agreed with Naseer et al. (2015), Vanisree et al. (2013) and Gour et al. (2017). Flag leaf width had negative and significant association with yield/hill. So, this trait should be discarded. Grain length and grain breadth have positive association with 1000 grain weight agreed with Ketan and Sarkar (2014). 
Table 4. Genotypic correlation coefficient $\left(\mathrm{r}_{\mathrm{g}}\right)$ and phenotypic correlation coefficient $\left(r_{p}\right)$ of selected 40 Boro rice germplasm

\begin{tabular}{|c|c|c|c|c|c|c|c|c|c|c|c|c|c|c|}
\hline & & $\begin{array}{l}\text { FLL } \\
(\mathrm{cm})\end{array}$ & $\begin{array}{l}\text { FLW } \\
(\mathrm{cm})\end{array}$ & DF & DM & $\begin{array}{c}\mathrm{PL} \\
(\mathrm{cm})\end{array}$ & ET & $\mathrm{HI}(\%)$ & FG/P & UFG/P & $\begin{array}{c}\text { GL } \\
(\mathrm{mm})\end{array}$ & $\begin{array}{c}\text { GB } \\
(\mathrm{mm})\end{array}$ & $\begin{array}{c}1000 \\
\text { gwt.(g) }\end{array}$ & $\begin{array}{c}\text { Yield } \\
\text { (kg/ha) }\end{array}$ \\
\hline \multirow{2}{*}{$\begin{array}{l}\mathrm{PH} \\
(\mathrm{cm})\end{array}$} & $r_{g}$ & $0.579^{* *}$ & $0.375^{* *}$ & 0.259 & 0.090 & $0.864 * *$ & 0.013 & -0.215 & 0.142 & 0.098 & $-0.299 *$ & $0.357^{*}$ & $-0.387^{*}$ & -0.109 \\
\hline & $r_{p}$ & $0.441 * *$ & 0.242 & 0.090 & -0.008 & $0.601 * *$ & 0.022 & -0.148 & 0.101 & 0.070 & -0.049 & 0.148 & -0.194 & -0.074 \\
\hline \multirow{2}{*}{$\begin{array}{l}\text { FLL } \\
(\mathrm{cm})\end{array}$} & $r_{g}$ & & $0.740 * *$ & 0.175 & 0.091 & $0.322 *$ & -0.430 ** & 0.100 & $0.290^{*}$ & 0.176 & -0.178 & $0.545^{* * *}$ & -0.148 & -0.096 \\
\hline & $r_{p}$ & & $0.602 * *$ & 0.095 & 0.018 & $0.317^{*}$ & $-0.350^{*}$ & 0.088 & 0.243 & 0.132 & -0.172 & 0.206 & -0.082 & -0.064 \\
\hline \multirow{2}{*}{$\begin{array}{l}\text { FLW } \\
(\mathrm{cm})\end{array}$} & $r_{g}$ & & & 0.071 & 0.018 & $0.428^{* *}$ & $-0.758^{* * *}$ & 0.044 & $0.290^{*}$ & 0.160 & 0.023 & 0.193 & -0.011 & $-0.325^{*}$ \\
\hline & $r_{p}$ & & & -0.026 & -0.084 & $0.340^{*}$ & $-0.610^{* * *}$ & 0.011 & 0.234 & 0.126 & 0.026 & 0.087 & -0.016 & -0.231 \\
\hline \multirow[t]{2}{*}{ DF } & $r_{g}$ & & & & $0.941 * *$ & $0.405^{* *}$ & 0.150 & $-0.317 *$ & $0.603 * *$ & k 0.199 & -0.108 & -0.216 & -0.293 & $0.507 * *$ \\
\hline & $r_{p}$ & & & & $0.612 * *$ & 0.103 & 0.082 & -0.184 & $0.317^{*}$ & 0.114 & -0.026 & -0.030 & -0.109 & 0.235 \\
\hline \multirow[t]{2}{*}{$\mathrm{DM}$} & $r_{g}$ & & & & & 0.208 & 0.147 & $-0.345^{*}$ & $0.541^{* *}$ & 0.279 & -0.003 & $-0.332^{*}$ & -0.148 & $0.353 *$ \\
\hline & $r_{p}$ & & & & & 0.090 & 0.132 & $-0.324 *$ & $0.465^{* *}$ & 0.244 & -0.021 & -0.122 & -0.136 & 0.266 \\
\hline \multirow{2}{*}{$\begin{array}{l}\mathrm{PL} \\
(\mathrm{cm})\end{array}$} & $r_{g}$ & & & & & & -0.252 & -0.263 & $0.436^{* *}$ & 0.229 & -0.255 & -0.102 & $-0.592 * *$ & -0.103 \\
\hline & $r_{p}$ & & & & & & -0.211 & -0.183 & $0.344^{*}$ & 0.172 & -0.185 & -0.106 & $-0.350 *$ & -0.055 \\
\hline \multirow[t]{2}{*}{ ET } & $r_{g}$ & & & & & & & $-0.286^{*}$ & -0.242 & -0.135 & -0.089 & -0.045 & -0.131 & 0.275 \\
\hline & $r_{p}$ & & & & & & & -0.238 & -0.235 & -0.131 & -0.046 & -0.048 & -0.115 & 0.264 \\
\hline \multirow[t]{2}{*}{ HI (\%) } & $r_{g}$ & & & & & & & & -0.009 & -0.197 & -0.122 & -0.072 & 0.099 & 0.138 \\
\hline & $r_{p}$ & & & & & & & & -0.002 & -0.185 & -0.115 & -0.015 & 0.079 & 0.115 \\
\hline \multirow[t]{2}{*}{$\mathrm{FG} / \mathrm{P}$} & $r_{g}$ & & & & & & & & & 0.030 & $-0.398^{*}$ & -0.244 & $-0.506 * *$ & $0.302 *$ \\
\hline & $r_{p}$ & & & & & & & & & 0.026 & $-0.322 *$ & -0.129 & $-0.399 *$ & 0.276 \\
\hline \multirow[t]{2}{*}{$\mathrm{UFG} / \mathrm{P}$} & $r_{g}$ & & & & & & & & & & 0.047 & -0.011 & -0.007 & -0.265 \\
\hline & $r_{p}$ & & & & & & & & & & 0.052 & -0.009 & -0.021 & -0.241 \\
\hline \multirow{2}{*}{$\begin{array}{l}\mathrm{GL} \\
(\mathrm{mm})\end{array}$} & $r_{g}$ & & & & & & & & & & & -0.064 & $0.875^{* *}$ & -0.166 \\
\hline & $r_{p}$ & & & & & & & & & & & -0.093 & $0.573 * *$ & -0.129 \\
\hline \multirow{2}{*}{$\begin{array}{l}\mathrm{GB} \\
(\mathrm{mm})\end{array}$} & $r_{g}$ & & & & & & & & & & & & $0.495^{* *}$ & -0.098 \\
\hline & $r_{p}$ & & & & & & & & & & & & 0.178 & -0.031 \\
\hline \multirow{2}{*}{$\begin{array}{l}1000 \\
\text { gwt. } \\
\text { (g) }\end{array}$} & $r_{g}$ & & & & & & & & & & & & & -0.056 \\
\hline & $r_{p}$ & & & & & & & & & & & & & -0.066 \\
\hline
\end{tabular}

$\mathrm{PH}=$ Plant height $(\mathrm{cm}), \mathrm{FLL}=$ Flag leaf length $(\mathrm{cm}), \mathrm{FLW}=$ Flag leaf width $(\mathrm{cm}), \mathrm{DF}=$ Days to flowering, $\mathrm{DM}=$ Days to maturity, $\mathrm{PL}=$ Panicle length $(\mathrm{cm}), \mathrm{ET}=$ Effective tiller, $\mathrm{HI}=$ Harvest index, $\mathrm{FG} / \mathrm{P}=\mathrm{No}$. of filled grain per plant, UFG/P= No. of unfilled grain per plant, $\mathrm{GL}=$ Grain length $(\mathrm{mm}), \mathrm{GB}=\mathrm{Grain}$ breadth $(\mathrm{mm}), 1000$ gwt. $=1000$ grain weight $(\mathrm{g})$

The path analysis revealed that the direct and indirect effect of different traits on yield at genotypic level. It was found that days to flowering, days to maturity and filled grain per panicle have positive direct effect on yield and made the total 
correlation positive and significant (Table 5) and agreed with Surek and Beser (2003). Effective tiller number and harvest index have high positive direct effect on yield/hill and made the total correlation positive which agreed with Ashok et al. (2016), Bornare et al. (2014), Moosavi et al. (2015) and Sandhya et al. (2014). So, direct selection based on these traits would be effective for the yield improvement of these boro germplasm. Flag leaf length, panicle length and 1000 grain weight have positive direct effect on yield but made the total correlation negative insignificant and agreed with Sandhya et al. (2014). So, selection based on these traits would not be effective for the breeders. Flag leaf width has high negative direct effect on yield/hill followed by unfilled grain per panicle, grain length, plant height and grain breadth which is agreed with Bhadru et al. (2012) and Devi et al. (2017). So, these traits should be discarded in direct selection method for yield improvement. The residual effect was found 0.565 , it means that $43.5 \%$ variability was estimated by 14 yield contributing traits of this study and $56.5 \%$ variability would be controlled by other yield contributing traits which were not in this study.

Table 5. Partitioning of genotypic correlation into direct (bold phase) and indirect components of selected 40 Boro rice germplasm

\begin{tabular}{|c|c|c|c|c|c|c|c|c|c|c|c|c|c|c|}
\hline & PH & $\begin{array}{c}\text { FLL(c } \\
\mathrm{m})\end{array}$ & $\begin{array}{c}\mathrm{FLW}(\mathrm{c} \\
\mathrm{m})\end{array}$ & DF & DM & $\begin{array}{c}\text { PL }(\mathrm{cm} \\
)\end{array}$ & ET & $\mathrm{HI}(\%)$ & FG/P & UFG/P & $\begin{array}{c}\mathrm{GL}(\mathrm{m} \\
\mathrm{m})\end{array}$ & $\begin{array}{c}\mathrm{GB}(\mathrm{mm} \\
)\end{array}$ & $\begin{array}{c}1000 \\
\text { gwt.(g) }\end{array}$ & $\begin{array}{c}\text { Y/hill } \\
\text { (g) }\end{array}$ \\
\hline PH & -0.255 & 0.101 & -0.089 & 0.038 & 0.008 & 0.205 & 0.005 & -0.037 & 0.031 & -0.022 & 0.039 & -0.019 & -0.114 & -0.109 \\
\hline $\begin{array}{l}\text { FLL } \\
(\mathrm{cm})\end{array}$ & -0.138 & 0.196 & -0.175 & 0.031 & 0.006 & 0.089 & -0.121 & 0.019 & 0.066 & -0.042 & 0.038 & -0.019 & -0.046 & -0.096 \\
\hline $\begin{array}{l}\text { FLW } \\
(\mathrm{cm})\end{array}$ & -0.089 & 0.134 & -0.256 & 0.005 & -0.013 & 0.108 & -0.232 & 0.006 & 0.065 & -0.038 & -0.005 & -0.005 & -0.005 & $-0.325^{*}$ \\
\hline DF & -0.042 & 0.025 & -0.005 & 0.235 & 0.079 & 0.066 & 0.034 & -0.048 & 0.203 & -0.039 & 0.014 & 0.058 & -0.073 & $0.507 * *$ \\
\hline DM & -0.012 & 0.012 & 0.009 & 0.178 & 0.106 & 0.044 & 0.043 & -0.067 & 0.124 & -0.069 & 0.022 & 0.018 & -0.055 & $0.353^{*}$ \\
\hline $\begin{array}{l}\mathrm{PL}(\mathrm{cm} \\
)\end{array}$ & -0.189 & 0.063 & -0.108 & 0.056 & 0.017 & 0.277 & -0.078 & -0.045 & 0.096 & -0.059 & 0.049 & 0.004 & -0.186 & -0.103 \\
\hline ET & -0.004 & -0.078 & 0.179 & 0.026 & 0.015 & -0.065 & 0.304 & -0.053 & -0.058 & 0.035 & 0.016 & 0.006 & -0.048 & 0.275 \\
\hline $\mathrm{HI}(\%)$ & 0.047 & 0.019 & -0.008 & -0.057 & -0.036 & -0.064 & -0.082 & 0.198 & -0.001 & 0.051 & 0.026 & 0.010 & 0.035 & 0.138 \\
\hline FG/P & -0.032 & 0.053 & -0.069 & 0.105 & 0.054 & 0.110 & -0.073 & -0.001 & 0.243 & -0.008 & 0.080 & 0.017 & -0.177 & $0.302 *$ \\
\hline UFG/P & -0.022 & 0.031 & -0.038 & 0.036 & 0.028 & 0.057 & -0.042 & -0.038 & 0.007 & -0.262 & -0.011 & 0.011 & -0.022 & -0.265 \\
\hline $\begin{array}{l}\mathrm{GL}(\mathrm{m} \\
\mathrm{m})\end{array}$ & 0.047 & -0.034 & -0.006 & -0.015 & -0.006 & -0.062 & -0.022 & -0.024 & -0.089 & -0.023 & -0.219 & 0.003 & 0.284 & -0.166 \\
\hline $\begin{array}{l}\mathrm{GB}(\mathrm{m} \\
\mathrm{m})\end{array}$ & -0.063 & 0.069 & -0.044 & -0.023 & -0.023 & -0.028 & -0.013 & -0.028 & -0.046 & 0.012 & 0.017 & -0.047 & 0.119 & -0.098 \\
\hline $\begin{array}{l}1000 \\
\text { gwt.(g) }\end{array}$ & 0.076 & -0.024 & 0.016 & -0.045 & -0.015 & -0.135 & -0.038 & 0.018 & -0.122 & 0.012 & -0.169 & -0.012 & 0.382 & -0.056 \\
\hline
\end{tabular}

\section{Residual Effect $=0.565$}

$\mathrm{PH}=$ Plant height $(\mathrm{cm}), \mathrm{FLL}=$ Flag leaf length $(\mathrm{cm}), \mathrm{FLW}=$ Flag leaf width $(\mathrm{cm}), \mathrm{DF}=$ Days to flowering, $\mathrm{DM}=$ Days to maturity, $\mathrm{PL}=$ Panicle length $(\mathrm{cm}), \mathrm{ET}=$ Effective tiller, $\mathrm{HI}=$ Harvest index, $\mathrm{FG} / \mathrm{P}=\mathrm{No}$. of filled grain per plant, $\mathrm{UFG} / \mathrm{P}=$ No. of unfilled grain per plant, $\mathrm{GL}=$ Grain length $(\mathrm{mm}), \mathrm{GB}=\mathrm{Grain}$ breadth $(\mathrm{mm}), 1000$ gwt. $=1000$ grain weight $(\mathrm{g})$ 
The summary of this finding is G5, G7, G19, G9 and G33 are the elite germplasm among these selected forty germplasm due to their overall performance in case of days to maturity, harvest index, effective tiller number and filled grain number.

\section{CONCLUSION}

Considering mean, range and other variability analysis this study expressed wide range of variability among 40 genotypes of selected Boro germplasm. The finding of this studyshows that G5 (Kamarang), G7 (Jamir), G19 (Boro135/1), G9 (Sadaboro) and G33 (Moghalsail) are the elite germplasm among these selected forty entries of Boro germplasm due to their overall better performance than average including days to maturity, harvest index, effective tiller number and filled grains per panicle. So, these entries in studied germplasm can be used in future breeding programs to create high yielding advance breeding lines with short maturity duration, profuse effective tillers along with more fertile filled grains per panicle and good harvest index so that the national rice yield could be increased and provide a safeguard to the national food security. However considering the findings of heritability, association and path analysis days to flowering, days to maturity and filled grain per panicle would be the selection criteria for further yield improvement

\section{ACKNOWLEDGMENT}

The authors would like to thank the Bangladesh Rice Research Institute authority to do this research.

\section{REFERENCES}

Ashok, S., Jyothula, D.P.B. and Ratnababu, D. (2016). Character association and path analysis for yield components and grain quality parameters of rice (Oryza sativa L.). International Journal of Agricultural Science and Research, 6(6): 253-258.

Babu Ravindra, V., Shreya, K., Singh, D.K., Usharani, G. and Siva Shankar, A. (2012). Correlation and path coefficient analysis studies in popular rice hybrids of India. International Journal of Scientific Research and Publication, 2(3): 1-5.

Bhadru, D., Mohan, Y.C., Rao, V.T., Bharathi, D. and Krishna, L. (2012). Correlation and path analysis studies in gall midge resistant cultures of rice (Oryza sativa L.). International Journal of Applied Biology and Pharmaceutical Technology, 3(2): 137140.

Bornare, S.S., Mittra, S.K. and Mehta, A.K. (2014). Genetic variability, correlation and path analysis of floral, yield and its component traits in CMS and restorer lines of rice (Oryza sativa L.). Bangladesh Journal of Botany, 43(1): 45-52.

Burton, G.W. (1952). Proceedings of Intercropping Grassland Congress: Quantities inheritance in grasses, 1(6): 277-283.

Chakravorty, A., Ghosh, P. and Sahu, P. (2013). Multivariate analysis of phenotypic diversity of landraces of rice of West Bengal. American Journal of Experimental Agriculture, 3(1): 110-123. 
Chuchert, S., Nualsri, C., Junsawang, N. and Soonsuwon, W. (2016). Genetic diversity, genetic variability, and path analysis for yield and its components in indigenous upland rice (Oryza sativa L. var. glutinosa). Songklanakarin Journal of Science and Technology, 323: 1-23

Comstock, R.E. and Robinson, H.F. (1952). Estimation of average dominance of genes. p. 494-516. In J. H. Gowen (ed.), Heterosis. Iowa State College Press, USA.

Devi, K.R., Chandra, B.S., Lingaiah, N., Hari, Y. and Venkanna, V. (2017). Analysis of variability, correlation and path coefficient studies for yield and quality traits in rice (Oryza sativa L.). Agricultural Science Digest, 37(1): 1-9.

Dewey, D.R. and Lu, K.H. (1959). A correlation and path coefficient analysis of components of crested wheat grass seed production. Agronomy Journal, 51(9): 515-518.

Doehlert, D.C., McMullen, M.S. and Hammond, J.J. (2001). Genotypic and environmental effects on grain yield and quality of oat grown in North Dakota. Crop Science, 41(4): 1066-1072.

Falconer, D.S. (1964). An Introduction to Quantitative Genetics. p. 312-324. In Oliver and Boyd ( $2^{\text {nd }}$ ed.), Edinburgh.

Gour, L., Koutu, G.K., Singh, S.K., Patel, D.D., Shrivastava, A. and Singh, Y. (2017). Genetic variability, correlation and path analyses for selection in elite breeding materials of rice (Oryza sativa L.) genotypes in Madhya Pradesh. The Pharma Innovation Journal, 6(11): 693-696.

Islam, M.Z., Khalequzzaman, M., Bashar, M.K., Ivy, N.A., Haque, M.M. and Mian, M.A.K. (2016). Variability assessment of aromatic and fine rice germplasm in Bangladesh based on quantitative traits. The Scientific World Journal. doi.org/10.1155/ 2016/2796720.

Johnson, H.W., Robinson, H.F., and Comstock, R.E. (1955). Estimates of genetic and implications in selection. Agronomy Journal, 50: 126-131.

Ketan, R. and Sarkar, G. (2014). Studies on variability, heritability, genetic advance and path analysis in some indigenous Aman rice (Oryza sativa L.). Journal of Crop and Weed, 10(2): 308-315.

Lush, J.L. (1949). Heritability of quantitative characters in farm animals. Heriditas, 35: 256261.

Mahmud, W. (2003). Bangladesh: development outcomes and challenges in the context of globalization. Paper presented at the conference on The Future of Globalization: Explorations in Light of Recent Turbulence co-sponsored by the Yale Center for the Study of Globalization and the World Bank, 10-11 Oct., 2003. Yale University.

Moosavi, M., Ranjbar, G., Zarrini, H.N. and Gilani, A. (2015). Correlation between morphological and physiological traits and path analysis of grain yield in rice genotypes under Khuzestan conditions. Biological Forum, 7(1): 43-47.

Naseer, S., Kashif, M., Ahmad, H.M., Iqbal, M.S. and Ali, Q. (2015). Estimation of genetic association among yield contributing traits in aromatic and non-aromatic rice (Oryza sativa L.) cultivars. Life Science Journal, 12(4s): 68-73.

Peyman, S. (2012). Diallel analysis to study genetic parameters of rice salinity tolerance traits at germination stage. SABRAO Journal of Breeding and Genetics, 44(1):42-57. 
Sadhya, Babu, G.S. and Kumar, R. (2014). Genetic variability, interrelationship and path analysis for yield improvement of rice genotypes. The Bioscan, 9(3): 1161-1164.

Surek, H. and Beser, N. (2003). Correlation and path coefficient analysis for some yieldrelated traits in rice (Oryza sativa L.) under thrace conditions. Turkish Journal of Agriculture and Foresrty, 27: 77-83.

Vanisree, S., Swapna, K., Raju, C.D., Raju, C.S. and Sreedhar, M. (2013). Genetic variability and selection criteria in rice. Journal of Biological and Scientific Opinion, 1(4): 341346.

Williams, P., Islam, M., Adomako, E., Raab, A., Hossain, S., Zhu, Y., Feldmann, J. and Meharg, A. (2006). Increase in rice grain arsenic for regions of Bangladesh irrigating paddies with elevated arsenic in groundwaters. Environmental Science and Technology, 40(16): 4903-4908.

Wright, S. (1921). Correlation and causation. Journal of Agricultural Research, 20: 557-585.

Ye, G., Collard, B., Zhao, X. and Nissila, E. (2013). Enhancing rice breeding efficiency: the role of breeding informatics. SABRAO Journal of Breeding and Genetics, 45(1): 143158. 